\title{
Stabilité thermomagnétoconvective d'un ferrofluide dans une couche poreuse en rotation
}

\author{
Thomas Desaive ${ }^{1, a}$, Marcel Hennenberg $^{2}$ et Pierre C. Dauby ${ }^{1}$ \\ 1 Université de Liège, Thermomécanique des Phénomènes Irréversibles, Allée du 6 août, 17, Bât. B5, 4000 Liège, Belgique \\ ${ }^{2}$ Université Libre de Bruxelles, MRC, Fac. des Sciences Appliquées, CP 165/62, Av. F. D. Roosevelt, 1050 Bruxelles, Belgique
}

Reçu le 15 décembre 2003, accepté le 10 février 2004

\begin{abstract}
Résumé - Dans ce travail, nous étudions la stabilité linéaire d'une couche poreuse en rotation saturée par un ferrofluide. Le système est placé dans le champ de pesanteur et chauffé par le bas ou par le haut. L'effet de la rotation est limité à la force de Coriolis et le milieu poreux est décrit par le modèle de Brinkman. Le problème aux valeurs propres linéaire est résolu par une méthode de Galerkin modifiée. Le comportement de la différence de température critique est discuté en fonction des différents paramètres du système pour la convection oscillante et la convection stationnaire.
\end{abstract}

Mots clés : Thermoconvection / milieu poreux / rotation

\begin{abstract}
Thermoconvective stability of a ferrofluid saturating a rotating porous layer. In this work, we study the linear stability of a rotating porous medium saturated with a ferrofluid. The system is in the gravity field and heated from below or from above. The effect of rotation is restricted to the Coriolis force and the porous medium is described by Brinkman's model. The linear eigenvalue problem is solved by means of a modified Galerkin method. The behavior of the critical temperature gradient is discussed in terms of various parameters of the system for both stationary and overstable convections.
\end{abstract}

Key words: Thermoconvection / porous medium / rotation

\section{Introduction}

L'étude de la convection d'un fluide dans une matrice poreuse en rotation est un problème non seulement d'intérêt fondamental mais aussi appliqué en raison des nombreuses applications industrielles et géophysiques. Dans ce travail, nous nous intéressons au cas où la matrice poreuse est saturée par un ferrofluide. Il s'agit d'une suspension colloïdale de nanoparticules magnétiques dans un liquide porteur (le plus souvent du kérosène). En l'absence de champ magnétique, les dipôles magnétiques des particules dans le fluide sont orientés de manière aléatoire en raison de l'agitation thermique et le fluide ne présente donc pas de magnétisation macroscopique. En présence d'un champ magnétique extérieur, les dipôles magnétiques s'alignent et une magnétisation du fluide est maintenant observable (Rosensweig [1], Berkovski et Bashtovoy [2]). L'étude de tels systèmes se justifie d'une part par les problèmes de recherches fondamentales qu'ils suscitent. D'autre part, les retombées technologiques et industrielles de ces recherches sont extrèmement séduisantes. Il suffit d'en citer les applications à la

\footnotetext{
a Auteur correspondant : tdesaive@ulg.ac.be
}

nanotechnologie (Zahn [3]) ou l'utilisation comme marqueurs pour suivre et guider des médicaments en cancérologie dans des os, exemples mêmes de milieux poreux (Alexion et al. [4]).

Ce travail a pour objet l'étude des instabilités thermoconvectives dans un milieu poreux saturé par un ferrofluide en présence de rotation. Le cas d'une couche liquide de ferrofluide en rotation a déjà été étudié par Auernhammer et Brand [5]. Le problème identique dans un milieu poreux a été étudié pour la première fois par Sekar et al. [6] avec un modèle de Darcy contenant un terme temporel puis avec un modèle de Brinkman (Sekar et Vaidyanathan [7]) pour des frontières libres et des conditions aux limites sur le potentiel magnétique simplifiées. Cette configuration n'est pas très physique mais présente l'avantage de pouvoir être étudiée analytiquement. Ces auteurs ont analysé le couplage avec le champ magnétique et calculé les seuils d'instabilité linéaire. D'autre part, le choix de leur adimensionalisation ne nous a pas paru très judicieux car il fait apparaître des termes proportionnels à $\sqrt{R a}$. Le nombre de Rayleigh doit donc toujours être positif ce qui élimine la possibilité d'un chauffage par le haut $(\Delta T<0 \rightarrow R a<0)$. 


\section{Formulation mathématique}

Soit un milieu poreux, d'extension horizontale infinie, en rotation à vitesse constante $\Omega$ autour d'un axe vertical et saturé par un ferrofluide. Ce système, d'épaisseur $d^{*}$, est sous l'influence d'un champ magnétique extérieur uniforme $\mathbf{H}_{\mathrm{e}}=H_{\mathrm{e}} \mathbf{e}_{z}$, dirigé suivant la verticale (Fig. 1).

\subsection{Bilan de quantité de mouvement}

Le milieu poreux est décrit par le modèle de Brinkman et la présence de ferrofluide implique d'ajouter une partie magnétique au tenseur des tensions visqueuses. Il est assez facile de montrer que la magnétisation $\mathbf{M}$ obéit à la distribution de Langevin (Rosensweig [1]). Pour des champs magnétiques forts, cas réalistes, on peut montrer que l'équation de Langevin se réduit à la relation linéaire suivante (Bashtovoy et al. [8], Stiles et Kagan [9], Blennerhasset et al. [10])

$$
\mathbf{M}=\left(M_{0}-\alpha_{\mathrm{pm}}\left(T-T_{0}\right)\right) \frac{\mathbf{H}}{H},
$$

le tenseur des tensions magnétique se simplifie considérablement et son gradient se réduit à un terme de pression plus la force magnétique de Kelvin

$$
\mathbf{F}_{\mathrm{m}}=\mu_{0} M \nabla \mathbf{H}
$$

où $\mu_{0}$ est la perméabilité magnétique du vide et $\mathbf{H}$ le vecteur champ magnétique.

Près de l'axe de rotation, nous pouvons négliger les effets centrifuges et limiter l'influence de la rotation au seul terme de Coriolis (Greenspan [11]). Le bilan de la quantité de mouvement est écrit en terme de la vitesse de filtration, ce qui explique la présence de la porosité $\phi$ dans le membre de gauche de (3). Le membre de droite contient l'ensemble des forces extérieures. Nous avons supposé que la description du milieu poreux obéissait à la loi phénoménologique de Brinkman qui complète la loi de Darcy habituelle par un terme dissipatif où apparaît une viscosité effective $\mu_{\mathrm{e}}$ dont la justification profonde est de permettre de raccorder la condition aux limites de la tension de cisaillement à l'interface fluide entre le milieu poreux et la phase de liquide pur. Le bilan de quantité de mouvement, dans des axes liés au système en rotation, est donc donné par l'équation suivante

$$
\begin{aligned}
& \rho_{\mathrm{l}}\left[\phi^{-1}\left(\partial_{\mathrm{t}} \mathbf{u}+2 \boldsymbol{\Omega} \wedge \mathbf{u}\right)+\phi^{-2}(\mathbf{u} \cdot \nabla) \mathbf{u}\right]= \\
& -\nabla \tilde{p}-\frac{\mu_{\mathrm{l}}}{K} \mathbf{u}+\mu_{\mathrm{e}} \nabla^{2} \mathbf{u}+\rho_{\mathrm{l}} \mathbf{g}+\mu_{0} M \nabla H
\end{aligned}
$$

où $\tilde{p}$ est une pression modifiée qui, outre la pression hydrostatique, contient l'ensemble des termes d'origine magnétique pouvant se mettre sous la forme d'un gradient (Rosensweig [1]), u est le vecteur vitesse de filtration, $\rho_{\mathrm{l}}$ la densité du ferrofluide, $\phi$ la porosité, $\mu_{1}$ la viscosité dynamique, $K$ la perméabilité du milieu poreux, g la pesanteur et $\mu_{\mathrm{e}}$ la viscosité effective apparaissant dans le modèle

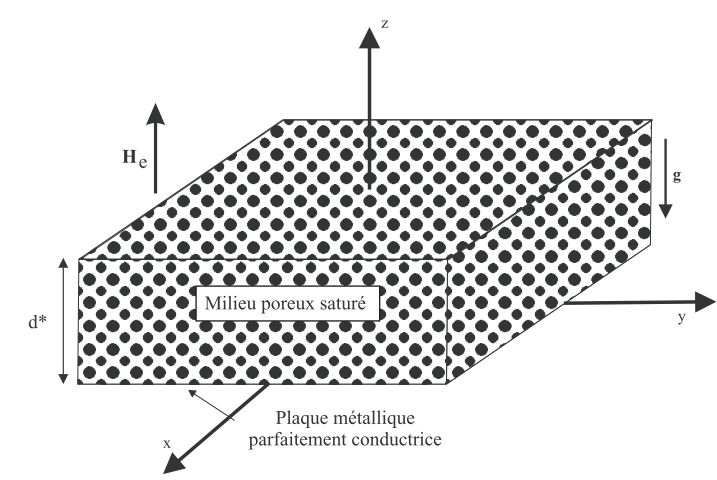

Fig. 1. Représentation schématique du système.

de Brinkman. Avant d'examiner le bilan d'énergie, il est intéressant de remarquer qu'il existe d'autres modèles de filtration comme ceux développés par Auriault et al. [12], Geindreau et Auriault [13] à partir d'une approche microscopique à l'échelle du pore.

\subsection{Bilan d'énergie}

La forme simple de l'équation d'état de la magnétisation permet d'écrire le bilan d'énergie sous la forme suivante (Stiles et Kagan [9], Blennerhasset et al. [10])

$$
(\rho c)_{\mathrm{p}} \partial_{\mathrm{t}} T+\left(\rho c_{\mathrm{P}}\right)_{\mathrm{f}} \mathbf{u} \cdot \nabla T=k_{\mathrm{p}} \nabla^{2} T
$$

où $(\rho c)_{\mathrm{p}}$ et $\left(\rho c_{\mathrm{P}}\right)_{\mathrm{f}}$ sont respectivement les capacités calorifiques du milieu poreux et du ferrofluide alors que $T$ est la température et $k_{\mathrm{p}}$ la conductivité thermique du milieu poreux. L'équation (4) devrait pour bien faire tenir compte de terme magnéto-diffusifs. Ceux-ci existent (Faynlayson [14]), mais leur contribution est en géneral négligeable pour la vaste majorité des ferrofluides habituels (Auernhammer et Brand [5], Berkovski et Bashtovoy [2], Huang et al. [15]). Par conséquent, la forme mathématique du bilan d'énergie est l'équation de Fourier conductive pour la matrice poreuse et l'équation de Fourier convective pour le fluide magnétique. Comme nous supposons la continuité locale de la température et du flux de chaleur le long du contact entre la matrice poreuse solide et le ferrofluide, l'équation (4) en résulte immédiatement.

\section{3 Étude de stabilité linéaire}

L'état de référence du système est un état de repos purement conductif, homogène horizontalement, caractérisé par une vitesse nulle et un profil de température linéaire. En le perturbant et après quelques calculs immédiats, on obtient aisément les équations linéarisées pour les variables perturbées. Sous forme adimensionnelle, les équations d'évolution linéaires des perturbations de la vitesse verticale $w$, de la composante verticale 
de la vorticité $\zeta$, de la température $T$ et du potentiel magnétique $\varphi$ s'écrivent finalement

$$
\begin{aligned}
\chi^{-1} \partial_{\mathrm{t}} \nabla^{2} w= & R a_{\mathrm{p}}(1+\mathcal{M}) \nabla_{\mathrm{h}}^{2} T+\Lambda D a \nabla^{4} w \\
& -\nabla^{2} w-\sqrt{T a} \partial_{z} \zeta-R a_{\mathrm{p}} \mathcal{M} \nabla_{\mathrm{h}}^{2} \partial_{z} \varphi \\
\chi^{-1} \partial_{\mathrm{t}} \zeta= & \Lambda D a \nabla^{2} \zeta-\zeta+\sqrt{T a} \partial_{z} w \\
\partial_{\mathrm{t}} T-w= & \nabla^{2} T \\
\nabla^{2} \varphi-\partial_{z} T= & 0
\end{aligned}
$$

où nous avons introduit les nombres sans dimensions suivants

$$
\begin{aligned}
R a_{\mathrm{p}} & =\frac{\alpha_{\mathrm{T}} g \rho_{0} \Delta T d^{*} K}{\mu_{\mathrm{l}} \kappa_{\mathrm{p}}}, \\
\chi & =\frac{\mu_{\mathrm{l}} d^{* 2} \phi}{\rho_{0} \kappa_{\mathrm{p}} K} \\
D a & =\frac{K}{d^{2}} \\
\Lambda & =\frac{\mu_{\mathrm{e}}}{\mu_{\mathrm{l}}} \\
T a & =\left(\frac{2 \Omega \rho_{0} K}{\mu_{\mathrm{l}} \phi}\right)^{2} \\
\mathcal{M} & =\frac{\mu_{0} \alpha_{\mathrm{pm}}^{2} \Delta T}{\rho_{0} \alpha_{\mathrm{T}} g d^{*}},
\end{aligned}
$$

respectivement le nombre de Rayleigh poreux, le nombre de Prandtl de filtration, le nombre de Darcy, le rapport des viscosités dynamiques, le nombre de Taylor poreux et le taux de magnétisation. Ce dernier se définit par le rapport du nombre de Kelvin poreux $(N=$ $\left.\frac{\mu_{0}\left(\alpha_{\mathrm{pm}} \Delta T\right)^{2} K}{\mu_{1} \kappa_{\mathrm{p}}}\right)$ et du nombre de Rayleigh poreux. Les parois sont supposées rigides, imperméables et parfaitement conductrices de la chaleur tandis que les conditions sur le potentiel magnétiques sont issues des relations de Maxwell (Fynlayson [14]). Nous effectuons ensuite la décomposition en modes normaux

$$
\left(\begin{array}{c}
w \\
\zeta \\
T \\
\varphi
\end{array}\right)=\left(\begin{array}{c}
W(z) \\
Z(z) \\
\Theta(z) \\
\Phi(z)
\end{array}\right) e^{i\left(a_{x} x+a_{y} y\right)+s t}
$$

où les amplitudes $W(z), Z(z), \Theta(z)$ et $\Phi(z)$ décrivent les variations inconnues par rapport à $z$ de la vitesse verticale, de la composante verticale de la vorticité, de la température et du potentiel magnétique. $\mathbf{a}=\left(a_{x}, a_{y}\right)$ désigne le nombre d'onde et $s$ représente le taux de croissance des perturbations. Le problème aux valeurs propres obtenu en introduisant (15) dans les équations (5)-(8) est résolu par une méthode Tau-Chebyshev afin d'obtenir les valeurs critiques du nombre de Rayleigh poreux et du nombre d'onde.
Les équations du problème linéaire se résument de la manière suivante :

$$
\begin{aligned}
\chi^{-1} s\left(D^{2}-a^{2}\right) W= & \Lambda D a\left(D^{2}-a^{2}\right)^{2} W \\
& -\left(D^{2}-a^{2}\right) W-\sqrt{T a} D Z \\
& +R a_{\mathrm{p}} \mathcal{M} a^{2} D \Phi-a^{2} R a_{\mathrm{p}}(1+\mathcal{M}) \Theta
\end{aligned}
$$

$$
\chi^{-1} s Z=\Lambda D a\left(D^{2}-a^{2}\right) Z-Z+\sqrt{T a} D W
$$

$$
\begin{aligned}
s \Theta & =\left(D^{2}-a^{2}\right) \Theta+W, \\
0 & =\left(D^{2}-a^{2}\right) \Phi-D \Theta,
\end{aligned}
$$

avec $D=\frac{\mathrm{d}}{\mathrm{d} z}$. Les conditions aux limites s'écrivent :

$$
\begin{aligned}
& W=D W=Z=\Theta=D \Phi-a \Phi=0, \text { en } z=0, \\
& W=D W=Z=\Theta=D \Phi+a \Phi=0, \text { en } z=1 .
\end{aligned}
$$

\section{Courbes neutres pour un chauffage par le bas}

\subsection{Frontières horizontales rigides}

Ce problème a été étudié par Venkatasubramanian (Venkatasubramanian et Kaloni [16]) dans le cas d'un ferrofluide en rotation mais le problème analogue dans une matrice poreuse n'a pas été analysé.

La figure 2 montre les courbes neutres correspondant à $\mathcal{M}=1, T a=10\left(D a=10^{-5}\right.$ et $\left.\Lambda=1\right)$. Les effets gravifique et magnétique sont d'amplitudes comparables et simultanément déstabilisants. En effet, alors que le mécanisme gravifique n'est déstabilisant que pour un chauffage par le bas (terme convectif proportionnel à $\Delta T$ ), le mécanisme magnétique est déstabilisant quel que soit le sens du gradient de température (terme convectif proportionnel à $\Delta T^{2}$ ). La présence de rotation dans le système implique que l'état marginal est tantôt stationnaire, tantôt oscillant selon les valeurs du nombre de Prandtl de filtration $\chi$. Il existe également une valeur de $\chi$ telle que les minima des courbes stationnaire et oscillante sont identiques. On parle alors de point de codimension 2. Pour caractériser la surstabilité, on examine la partie imaginaire du taux de croissance $\left(s_{i}\right)$ pour avoir la fréquence des oscillations. Cependant, c'est encore et toujours $R a_{\mathrm{p}}$ qui donne la contrainte minimale, c'est-à-dire la différence de température au-delà de laquelle l'instabilité apparaît. La figure 3 donne l'évolution de $s_{i}$ en fonction du nombre d'onde, pour deux valeurs du nombre de Prandtl de filtration. Elle passe par un maximum avant de retomber à zéro pour les grands nombres d'onde sauf pour les faibles valeurs de $\chi$ où elle décroît continûment vers zéro. 


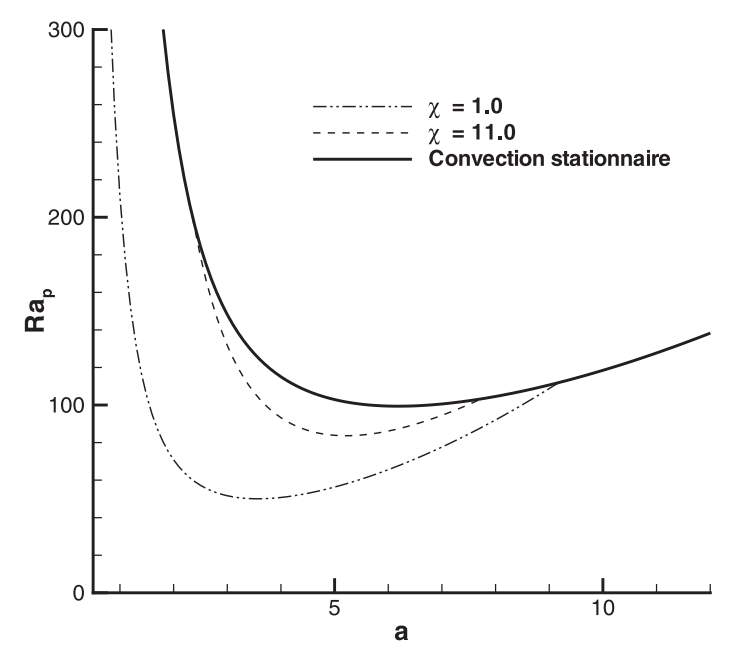

Fig. 2. Courbes de stabilité marginale pour $D a=10^{-5}$, $T a=10$ et $\mathcal{M}=1$. Les courbes en pointillés représentent la convection oscillante et les courbes pleines la convection stationnaire.

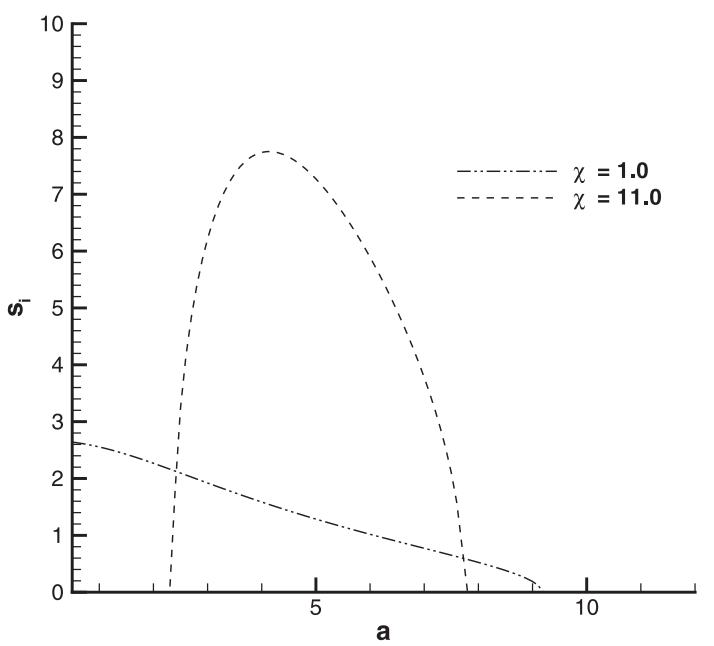

Fig. 3. Fréquence associée au mode oscillant en fonction du nombre d'onde pour $D a=10^{-5}, T a=10$ et $\mathcal{M}=1$.

\section{Ferroconvection pour un chauffage par le haut}

\subsection{Courbes neutres}

Nous considérons maintenant le cas où le système est chauffé par le haut. Définissons le paramètre suivant

$$
R_{\mathrm{p}}=-R a_{\mathrm{p}}, \quad(\mathcal{M}<0)
$$

qui représente une grandeur positive lors d'un chauffage par le haut.

La figure 4 montre l'évolution de $R_{\mathrm{p}}$ en fonction du nombre d'onde pour $T a=10$. On observe sur ces courbes de nombreuses discontinuités ainsi que plusieurs minima locaux aussi bien oscillants que stationnaires. Ces discontinuités proviennent du fait que nous avons représenté les états de stabilité marginale c'est-à-dire les plus petites valeurs de $R_{\mathrm{p}}$ donnant lieu à une instabilité.

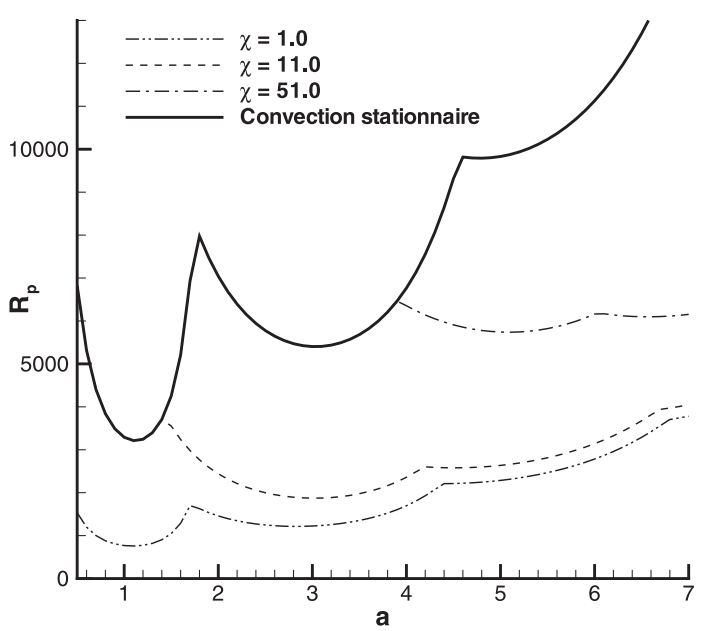

Fig. 4. Courbes de stabilité marginale pour $D a=10^{-5}$, $T a=10$ et $\mathcal{M}=-2$. Les courbes en pointillés représentent la convection oscillante et les courbes pleines la convection stationnaire.

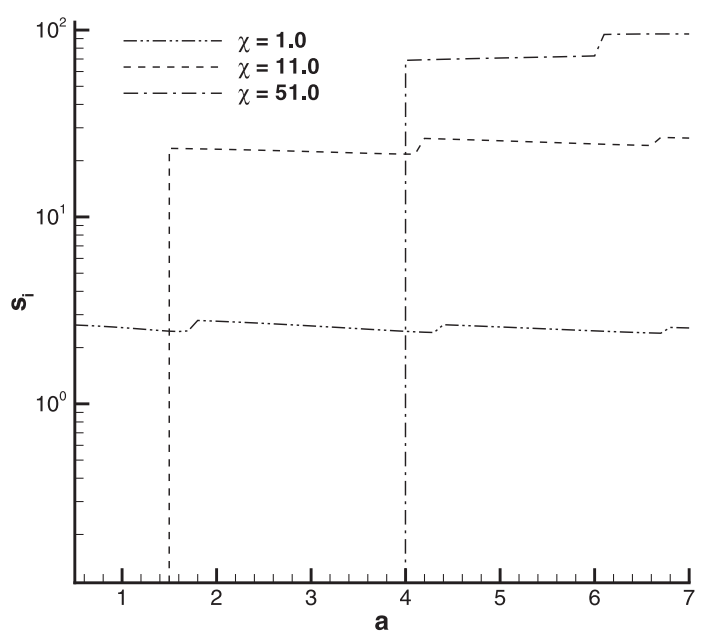

Fig. 5. Fréquence associée au mode oscillant en fonction du nombre d'onde pour $D a=10^{-5}, T a=10$ et $\mathcal{M}=-2$.

Le problème aux valeurs propres générant un nombre infini de valeurs propres par nombre d'onde, il se peut que pour deux nombres d'ondes voisins, ce ne soit pas la même valeur propre qui bifurque la première. En d'autres termes, nous observerons une structuration verticale différente suivant la valeur du nombre d'onde. Afin de mieux comprendre l'origine de ces discontinuités, nous avons tracé sur la figure 6 la courbe de stabilité marginale stationnaire correspondant à $D a=10^{-5}, \mathcal{M}=-2$ et $T a=10$. Pour trois valeurs du nombre d'onde $a$, nous avons représenté la fonction de courant. On voit ainsi que lorsque $a=1$, la structuration verticale la plus stable correspond à une seule cellule. Lorsque $a=3$, la structuration verticale la plus stable comporte maintenant deux cellules et puis trois pour $a=6$. Le raisonnement que nous venons de tenir pour justifier les discontinuités dans les courbes neutres reste valable pour expliquer les discontinuités présentes dans les courbes d'évolution de la fréquence des solutions oscillantes (Fig. 5). 


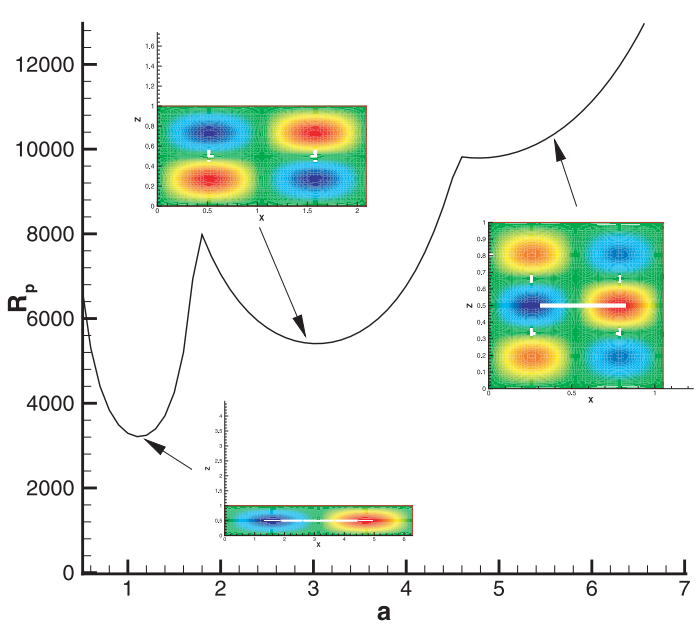

Fig. 6. Allure de la fonction de courant en plusieurs points de la courbe neutre stationnaire correspondant à $D a=10^{-5}$, $\mathcal{M}=-2.0$ et $T a=10$.

Comme nous pouvions nous y attendre, les valeurs du seuil de convection sont beaucoup plus élevées que dans le cas du chauffage par le bas. En effet, seuls les effets magnétiques tendent à déstabiliser le système alors que les effets gravifiques le stabilisent; il faudra fournir une beaucoup plus grande quantité d'énergie pour que les effets déstabilisants l'emportent et que l'instabilité apparaisse.

Notre message revient en fait à élargir à une matrice poreuse la conclusion de Blums et al. [17] à la page 207, « the temperature perturbations of the magnetizing field always lead to the thermoconvective fluid destabilization ». L'explication en est assez simple, $R a_{\mathrm{p}}$ et $\mathcal{M}$ étant tous deux de même signe quelque soit la direction de chauffage, leur produit est toujours positif. Ceci n'a pu être observé par Fynlayson [14] qui introdut une adimensionnalisation basée sur $\sqrt{R a}$; cette racine devant toujours être celle d'un nombre positif, il ne considère que le chauffage par le haut.

\section{Conclusions}

Dans ce travail, nous avons examiné la stabilité d'une couche poreuse en rotation saturée par un ferrofluide. Deux mécanismes de convection sont présents : l'un gravifique et l'autre magnétique. Le premier de nature déstabilisante lorsque le système est chauffé par le bas alors que le second est déstabilisant quelle que soit le sens du gradient thermique. Ce système pourra donc toujours exhiber de la convection et ce, même en conditions de microgravité.

Lorsque le système est chauffé par le bas, les effets gravifique et magnétique s'additionnent pour donner lieu à une instabilité de Rayleigh plus intense car ces deux effets sont simultanément déstabilisants. Le point critique est soit stationnaire soit oscillant suivant la valeur du nombre de Prandtl de filtration.

Nous avons terminé cette étude par l'analyse du chauffage par le haut qui n'avait jamais été envisagé auparavant dans un milieu poreux. Nous avons montré que les courbes neutres avaient une allure très différente de celle des courbes obtenues pour le chauffage par le bas. Les courbes neutres, qu'elles soient stationnaires ou oscillantes présentent une succession de minima secondaires dont l'origine a été élucidée par le tracé des fonctions de courant correspondantes. Nous avons pu montrer que ces différents minima étaient caractéristiques d'une structuration verticale (i.e. un nombre d'onde vertical) différente.

\section{Références}

[1] R.E. Rosensweig, Ferrohydrodynamics, Cambridge University Press, Cambridge, 1985

[2] Y. Berkovski, B. Bashtovoy (ed.), Magnetic Fluids and Applications Handbook, Begell House, New York, 1998

[3] M. Zahn, Magnetic fluid and nanoparticle applications to nanotechnology, J. Nanoparticle Res. 31 (2001) 73-78

[4] C. Alexion, R. Schmid, R. Jurgons, C. Bergemann, W. Arnold, F.G. Parak, Targeted turnor therapy with magnetic drug targeting. Therapeutic efficacy of ferrofluid bound mitoscantrone, in S. Odenbach (ed.), Lecture Notes in Physics, Springer-Verlag, 2002

[5] G.K. Auernhammer, H.R. Brand, Thermal convection in a rotating layer of magnetic fluid, Eur. Phys. J. B 16 (2000) 157-168

[6] R. Sekar, G. Vaidyanathan, A. Ramanathan, The ferroconvection in fluids saturating a rotating densely packed porous medium, Int. J. Engng Sc. 31(2) (1993) 241-250

[7] R. Sekar, G. Vaidyanathan, Convective instability of a magnetized ferrofluid in a rotating porous medium, Int. J. Engng Sc. 31(8) (1993) 1139-1150

[8] B. Bashtovoy, Y. Berkovski, A.N. Vislovich, Introdution to thermomechanics of Magnetic Fluids, Hemisphere Publishing Corporation, 1988

[9] P.J. Stiles, M. Kagan, Thermoconvective instability of a ferrofluid in a strong magnetic field, J. of Colloid and Inter. Sci. 134 (1989) 435-448

[10] P.J. Blennerhasset, F. Lin, P.J. Stiles, Heat transfer through strongly magnetized ferrofluids, Proc. R. Soc. London A 433 (1991) 165-177

[11] H.P. Greenspan, The Theory of Rotating Fluids, Cambridge University Press, UK, 1968

[12] J.-L. Auriault, C. Geindreau, P. Royer, Filtration Law in rotating porous media, Comptes rendus de l'Académie des sciences, Série IIb, Mécanique 328(11) (2000) 779-784

[13] C. Geindreau, J.-L. Auriault, Magnetohydrodynamics flow through porous media. Comptes rendus de l'Académie des sciences - Série IIb - Mécanique 329(6) (2001) 445-450

[14] B.A. Fynlayson, Convective instability of ferromagnetic fluids, J. Fluid Mech. 40 (1970) 753

[15] J. Huang, B.F. Edwards, D. Gray, Thermoconvective instability of paramagnetic fluids in a uniform field, Phys. Fluids 9 (1997) 1819-1825

[16] S. Venkatasubramanian, P.N. Kaloni, Effects of rotation on the thermoconvective instability of a horizontal layer of ferrofluids, Int. J. Engng Sci. 32(2) (1994) 237-256

[17] E. Blums, A. Cebers, M.M. Maiorov, Magnetic Fluids, Watter de Gruyter, Berlin, New York 